\title{
Optical side-band generation in THz Fabry-Perot laser cavities
}

DOI:

10.1063/1.5001334

Document Version

Accepted author manuscript

Link to publication record in Manchester Research Explorer

\section{Citation for published version (APA):}

Folland, T., Hua, L., Marshall, O., Khairuzzaman, M., Beere, H. E., Ritchie, D. A., \& Chakraborty, S. (2017). Optical side-band generation in THz Fabry-Perot laser cavities. Applied Physics Letters. https://doi.org/10.1063/1.5001334

\section{Published in:}

Applied Physics Letters

\section{Citing this paper}

Please note that where the full-text provided on Manchester Research Explorer is the Author Accepted Manuscript or Proof version this may differ from the final Published version. If citing, it is advised that you check and use the publisher's definitive version.

\section{General rights}

Copyright and moral rights for the publications made accessible in the Research Explorer are retained by the authors and/or other copyright owners and it is a condition of accessing publications that users recognise and abide by the legal requirements associated with these rights.

\section{Takedown policy}

If you believe that this document breaches copyright please refer to the University of Manchester's Takedown Procedures [http://man.ac.uk/04Y6Bo] or contact uml.scholarlycommunications@manchester.ac.uk providing relevant details, so we can investigate your claim.

\section{OPEN ACCESS}




\title{
Optical side-band generation in THz Fabry-Perot laser cavities
}

\author{
Thomas G. Folland ${ }^{1}$, Li Hua ${ }^{1}$, Owen P. Marshall ${ }^{1}$, Md. Khairuzzaman ${ }^{1}$, Harvey E. Beere ${ }^{2}$, David A. \\ Ritchie $^{2}$ and Subhasish Chakraborty ${ }^{1, a)}$
}

${ }^{1}$ School of Electrical and Electronic Engineering, University of Manchester, Manchester, M13 9PL, UK

${ }^{2}$ Cavendish Laboratory, Department of Physics, University of Cambridge, Cambridge CB3 0HE, UK

a) Electronic mail: s.chakraborty@manchester.ac.uk

Optical nonlinearities in semiconductor laser cavities can be exploited to characterize the properties of laser radiation or perform high speed frequency conversion operations. For example, nonlinear upconversion inside the cavity of quantum cascade lasers allows the use of near infrared optical components to measure high-speed terahertz or mid-infrared optical effects. This letter investigates two aspects of cavity up-conversion which control both the bandwidth and up-converted power; waveguide dispersion and cavity feedback. Specifically, we up-convert multi-mode Fabry Perot terahertz laser emission and detect each THz mode as a sideband signal on an optical carrier in the near infrared. Analysis of these results shows that a single frequency near infrared laser can up-convert terahertz modes spanning a bandwidth of approximately $220 \mathrm{GHz}$; limited by the group index mismatch between the near infrared and terahertz waves. Secondly, transfer matrix techniques are used to study strong cavity feedback on all three waves, which produces etalon-like resonances in the sideband power. This can significantly enhance the efficiency of the conversion process, in agreement with experiments. It is thus possible to achieve high up-conversion efficiency in quantum cascade lasers for both characterizing broadband laser sources and performing frequency conversion in the near infrared. 
25 The high optical power inside semiconductor laser cavities makes them an appealing medium for nonlinear optics. Possible applications include nonlinear frequency conversion [1,2], generation of terahertz (THz) light $[3,4]$, and mixing of THz radiation with near infrared (NIR) waves for detection using telecoms components [5-9]. However, nonlinear optics in semiconductor lasers comes with two design challenges: waveguide dispersion and facet feedback. Firstly, phase matching (PM) in nonlinear processes is highly sensitive to the refractive indices experienced by the waves involved [10,11]. Therefore, the combined material and waveguide dispersion of the laser cavity controls both the frequency and bandwidth of the nonlinear process. If these are not well understood then serious limitations are placed on the effectiveness of the conversion technique. Secondly, the use of a resonant laser cavity also induces feedback on pump, idler, and signal waves, thereby altering the efficiency of nonlinear processes $[12,13]$. Indeed, when the frequencies of the mixed waves are also resonant with the laser cavity it may be possible to significantly enhance the conversion efficiency. This letter examines the effect of both dispersion and cavity feedback in the up-conversion of THz waves to the NIR within the cavity of a quantum cascade laser (QCL). Crucially, we show that waveguide dispersion allows simultaneous up-conversion of THz frequencies spanning $220 \mathrm{GHz}$, and that the nonlinear process is resonantly enhanced by cavity feedback.

Using cavity up-conversion to detect $\mathrm{THz}$ signals in the NIR is appealing as it avoids the challenges of direct detection in the far infrared $[2,5,6,14,15]$. In THz QCLs we can exploit the large $\chi^{(2)}$ nonlinear susceptibility of the GaAs active region for intra-cavity up-conversion. The lack of birefringence makes achieving PM (equation 1) between pump $\left(f_{\mathrm{p}}\right)$, idler $\left(f_{\mathrm{i}}\right)$, and signal waves $\left(f_{\mathrm{s}}=f_{\mathrm{p}} \pm f_{\mathrm{i}}\right)$ difficult [10];

$$
n_{\mathrm{s}} f_{\mathrm{s}}=n_{\mathrm{p}} f_{\mathrm{p}} \pm n_{\mathrm{i}} f_{\mathrm{i}}
$$

However, by exploiting a combination of the anomalous dispersion introduced by the Reststrahlen band and modal phase matching [16], one can achieve efficient PM between THz and NIR waves $\left(f_{\mathrm{p}}=f_{\mathrm{NIR}}, f_{\mathrm{i}}=\right.$ $f_{\mathrm{THz}}$ ) [11]. In practice, this is accomplished by integrating a NIR waveguide into the active region of the 
QCL, leading to a strong optical field overlap and hence high conversion efficiency $[2,5,6]$. The result is conversion of the THz wave into a NIR sideband signal. The sideband encodes information about any temporal variations in the frequency, amplitude and phase of the THz wave, allowing for coherent characterisation of QCLs with high-responsivity telecoms components [7-9]. Here, multi-frequency emission from a Fabry Perot (FP) THz QCL is up-converted to the NIR, which allows measurement of the up-conversion properties of each THz mode simultaneously.

55 Our results show that each $\mathrm{THz}$ mode of the FP laser has a distinctly measurable NIR frequency where the three waves are phase matched and all the process produces the most power (the so-called phase matching point). This is surprising given the relatively small $\mathrm{THz}$ mode spacing $(\sim 8 \mathrm{GHz})$. By measuring the changes in $f_{\mathrm{THz}}$ and associated phase matched $f_{\mathrm{NIR}}$, we can calculate that the up-conversion process spans a frequency range of $\sim 220 \mathrm{GHz}$. This bandwidth is ultimately limited by the large difference in group indices between $\mathrm{THz}$ and sideband signals, which could in principle be managed using waveguide dispersion engineering. Additionally, a transfer matrix model (TMM) of the nonlinear process is used to study etalon fringes present in the up-converted sideband power. These fringes are a consequence of FP feedback on the NIR waves (both pump and signal), and significantly enhances the conversion efficiency. This combination of experiment and simulation provides a complete picture of up-conversion in FP laser cavities, and suggests that up-conversion in QCLs can be broadband with moderate efficiency. Our work opens a route to characterisation of broadband mode locked THz QCLs, as well as applications in frequency conversion for both multicasting and de-multiplexing of communication signals [17].

Two QCL ridges, with a $12.8 \mu \mathrm{m}$-high $\mathrm{GaAs} / \mathrm{Al}_{0.15} \mathrm{Ga}_{0.85} \mathrm{As}$ bound-to-continuum active region, containing an integrated NIR guiding layer, were fabricated on a single chip [5,18]. These two $200 \mu \mathrm{m}$-wide semiinsulating surface plasmon (SI-SP) waveguides were cleaved to form $\sim 4.6 \mathrm{~mm}$ long laser cavities. The choice of SI-SP waveguides (as opposed to double-metal waveguides) significantly simplified device fabrication. Devices were operated in pulsed mode (repetition rate $f_{\text {rep }}$, peak current $I_{\mathrm{QCL}}$ ), well above threshold and at a heatsink temperature of $10 \mathrm{~K}$. Current pulses were provided by an Agilent 8144A pulse 
generator, along with an Avtech AVX-MRB2 pulse amplifier. Both QCLs showed similar THz emission properties and were characterized with a Bruker Vertex 80 Fourier transform infrared (FTIR) spectrometer and a calibrated thermopile (placed directly in front of a laser facet) while mounted in a Janis ST100 cryostat. The fiber optic setup used to generate and detect QCL radiation in the NIR is illustrated in Figure 1(a) [7,18]. NIR light was generated by a tunable external cavity laser (Yenista OSICS T100 - power $3 \mathrm{~mW}$ ) and injected into the NIR waveguide embedded within the QCL [5] via a single mode optical fiber (SMF) butt-coupled to a QCL facet inside a Janis ST500 micromanipulation cryostat. Fiber to QCL input/output coupling losses are approximately $21 \mathrm{~dB}$. THz light was generated inside the electrically driven QCL, which subsequently mixed with the injected NIR wave, generating sum and difference frequency sidebands. To optimize the sideband intensity the injected NIR light was TE polarized, with a NIR laser wavelength chosen to lie close to the PM point of the up-conversion process $(\sim 1.3 \mu \mathrm{m})$. The NIR sidebands were collected alongside the main NIR pump mode using a second butt-coupled SMF (at the other QCL facet) and detected using an optical spectrum analyzer (OSA) (Yokogawa AQ6370C).

Our simulations of the nonlinear properties of this system were based on a nonlinear TMM method $[19,20]$. This extends our previous linear transfer matrix techniques (which use the Fresnel coefficients for each of the three waves) [21] by introducing a second-order polarization $P^{\mathrm{NL}}=2 \chi^{(2)} E_{\mathrm{p}} E_{\mathrm{i}}$ and solving the coupled wave equations for the signal $\left(E_{\mathrm{s}}\right)$ generated by the pump $\left(E_{\mathrm{p}}\right)$ and idler $\left(E_{\mathrm{i}}\right)$ fields. First, linear propagation matrices are defined for the pump and idler waves using a conventional transfer matrix in terms of input and output waves:

$$
\left(\begin{array}{l}
E_{u t}^{+} \\
E_{u t}^{-}
\end{array}\right)=\mathbf{T}_{u}\left(\begin{array}{c}
E_{u 0}^{+} \\
E_{u 0}{ }^{-}
\end{array}\right)
$$

where $\mathbf{T}_{\mathrm{u}}\left(\mathrm{u}=\mathrm{p}\right.$, i representing pump and idler waves respectively) is a $2 \times 2$ matrix for the electric field $E_{\mathrm{u}}$ within the FP cavity. The components $\left(T_{\mathrm{u}(\mathrm{ji})}\right)$ of this matrix define reflection $\left(\rho_{\mathrm{u}}=-T_{\mathrm{u}(21)} / T_{\mathrm{u}(22)}\right)$. For $E_{\mathrm{s}}$ the 
transfer matrix includes two terms; one describing propagation through the layer analogous to $\mathbf{T}_{\mathrm{u}}$, the second describing the generated field as per the nonlinear wave equation:

$$
\left(\begin{array}{l}
E_{s t}^{+} \\
E_{s t}^{-}
\end{array}\right)=\mathbf{T}_{s}\left(\begin{array}{c}
E_{s 0}^{+} \\
E_{s 0}^{-}
\end{array}\right)+\left(\begin{array}{l}
r^{+} \\
r^{-}
\end{array}\right)
$$

In equation $3, E_{\mathrm{st}}$ and $E_{\mathrm{s} 0}$ are the plane wave coefficients at the output and input, the sign $+(-)$ denotes the forward (backward) propagating wave. $\mathbf{T}_{\mathrm{s}}(2 \times 2)$ defines the linear transfer matrix of $E_{\mathrm{s}}$, whereas $r^{+}$and $r^{-}$ define the fields generated in the layer due to the mixing of $E_{\mathrm{p}}$ and $E_{\mathrm{i}}$. Detailed expressions for $r^{+}$and $r$ are described in references $[19,20]$. From equation (3) it is possible to derive expressions for the output efficiency $\left(\eta_{t}=\left(E_{\mathrm{st}}{ }^{+}\right)^{2} /\left(E_{\mathrm{p} 0}{ }^{+}\right)^{2}\right)$ in terms of the incident pump and idler waves [19,20]:

105

$$
\eta_{t}=\left[-\frac{T_{s(12)}}{T_{s(22)}}\left(M_{12}^{+}+M_{12}^{-}\right)+\left(M_{11}^{+}+M_{11}^{-}\right)\right] \frac{I_{i}}{A}
$$

Here $P_{\mathrm{i}}$ is the power of the idler wave, $A$ is the effective cross section area, and $\mathbf{M}^{+}\left(\mathbf{M}^{-}\right)$is a $2 \times 1$ nonlinear vector with elements $M_{11}{ }^{+}$and $M_{12}{ }^{+}\left(M_{11}{ }^{-}\right.$and $\left.M_{12}{ }^{-}\right)$, defined by:

$$
\mathbf{M}^{ \pm}=\left(-\frac{2 k_{s 0}{ }^{2} \chi^{(2)}}{k_{s n}{ }^{2}-\left(k_{p n} \pm k_{i n}\right)^{2}}\right)\left(G_{0}^{-1} G_{p \pm i} Q_{p \pm i}-G_{0}^{-1} G_{s} Q_{s} G_{s}^{-1} G_{p \pm i}\right)\left(\begin{array}{c}
\left(T_{p 11}+\rho_{p} T_{p 12}\right)\left(T_{i 11}+\rho_{i} T_{i 12}\right) \\
\left(T_{p 21}+\rho_{p} T_{p 22}\right)\left(T_{i 21}+\rho_{i} T_{i 22}\right)
\end{array}\right)
$$

In equation (5) $k_{\mathrm{s} 0}$ is the free-space signal wave wavenumber, whereas $k_{\mathrm{pn}}, k_{\mathrm{in}}$ and $k_{\mathrm{sn}}$ are the pump, idler and signal wavenumbers within the cavity. Finally $G_{0}, G_{\mathrm{s}}, G_{\mathrm{p}+\mathrm{i}}$ and $G_{\mathrm{p}-\mathrm{i}}$ are scattering matrices and $Q_{\mathrm{s}}$, $Q_{\mathrm{p}+\mathrm{i}}$ and $Q_{\mathrm{p}-\mathrm{i}}$ are phase matrices defined as:

$$
\begin{gathered}
G_{0}=\left(\begin{array}{cc}
1 & 1 \\
n_{0} & -n_{0}
\end{array}\right), G_{s}=\left(\begin{array}{cc}
1 & 1 \\
k_{s n} / k_{s 0} & -k_{s n} / k_{s 0}
\end{array}\right), G_{p \pm i}=\left(\begin{array}{cc}
1 & 1 \\
\left(k_{p n} \pm k_{i n}\right) / k_{s 0} & -\left(k_{p n} \pm k_{i n}\right) / k_{s 0}
\end{array}\right) \\
Q_{s}=\left(\begin{array}{cc}
\exp \left(j k_{s n} L\right) & 0 \\
0 & -\exp \left(j k_{s n} L\right)
\end{array}\right), Q_{p \pm i}=\left(\begin{array}{cc}
\exp \left(j\left(k_{p n} \pm k_{i n}\right) L\right) & 0 \\
0 & -\exp \left(j\left(k_{p n} \pm k_{i n}\right) L\right)
\end{array}\right)
\end{gathered}
$$


We assume that the length of the cavity is $L=4.6 \mathrm{~mm}$, the injected $\mathrm{THz}$ power $P=40 \mathrm{~mW}$, the effective

115 mode overlap $1 / A=4 \times 10^{8} \mathrm{~m}^{-2}[6]$, and the second order nonlinear susceptibility $\chi^{(2)}$ of GaAs is 100

$\mathrm{pm} / \mathrm{V}[10]$. Unlike in previous work, the transfer matrix approach accounts for cavity enhancement of the electromagnetic field, so the measured THz power (Fig. 1(b)) could be used as the input for simulations. Simulations use the refractive index of bulk GaAs from reference [22], with a small offset $(-0.00065)$ to shift the PM wavelength from $1.1 \mu \mathrm{m}$ to $1.3 \mu \mathrm{m}$, as observed experimentally. To account for the effect of

120 material gain on the terahertz wave dispersion [23], we estimated a Lorentzian gain profile with full width half maximum of $200 \mathrm{GHz}$, and a peak gain product of $\mathrm{gL}=1$ at $2.85 \mathrm{THz}$. This difference between simulation and experiment arises from the presence of free carriers, waveguide dispersion and cryogenic operation in the QCL, all of which change the refractive index of the active region.
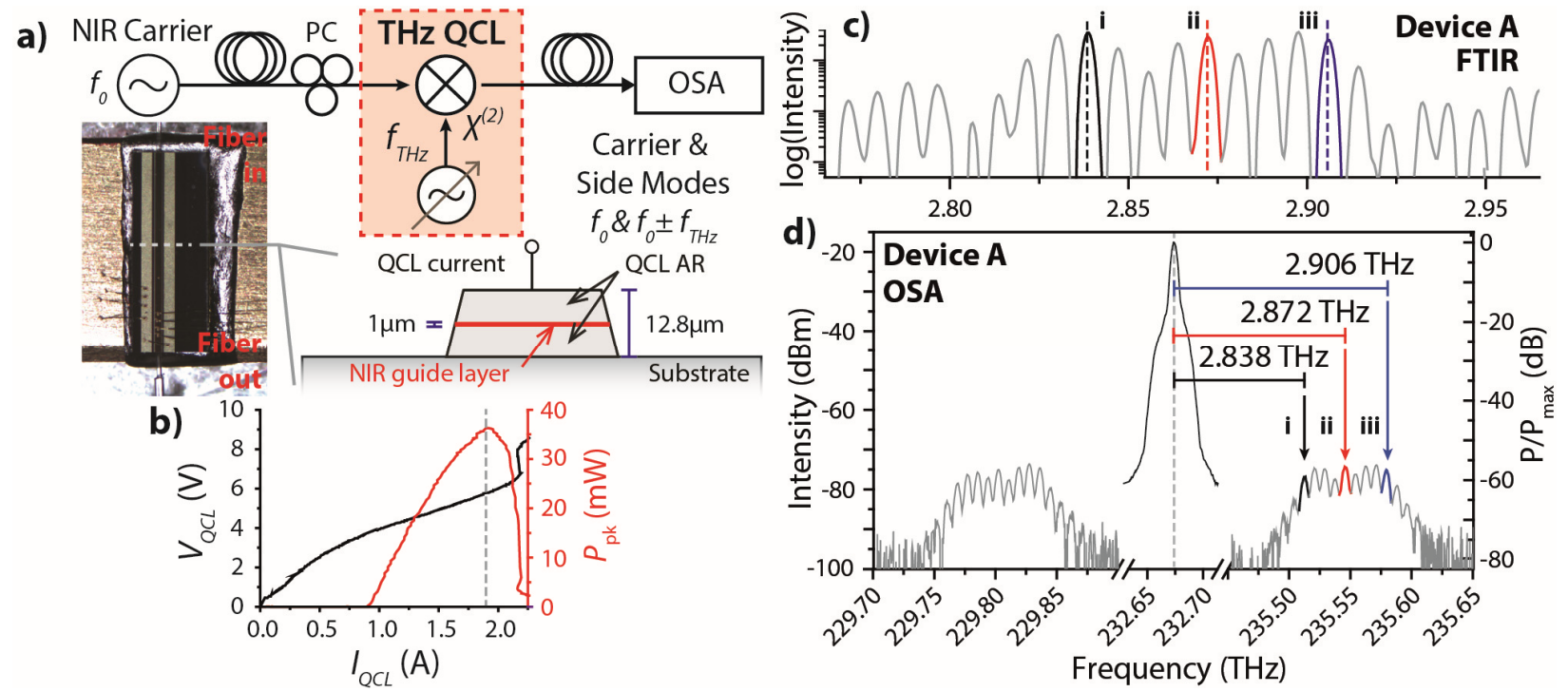

125 Figure 1. Up-conversion of THz waves to the NIR. a) the experimental setup and a schematic of the laser ridge, b) electrical and THz output power behaviour of the QCL, c) a FTIR spectrum of the QCL at $I_{Q C L}=1870 \mathrm{~mA}$ and d) the experimentally measured optical spectrum in the OSA. Highlighted are the three modes analyzed in Figure 2.

Figures 1(b) and 1(c) respectively show the current-voltage curve and multimode emission spectrum of Device A $\left(I_{\mathrm{QCL}}=1850 \mathrm{~mA}\right.$, duty cycle $\left.=10 \%\right)$, the latter measured directly by FTIR. Figure $1 \mathrm{~d}$ shows the optical spectrum collected from the OSA when the THz signal is up converted to the NIR $\left(I_{\mathrm{QCL}}=\right.$ 
$1870 \mathrm{~mA}$, duty cycle $=15 \%$ ). This spectrum shows the NIR carrier wave (pump) and the up-converted THz sideband (signal). The sideband shows the up-converted multi-mode emission of the QCL, with a maximum conversion efficiency of $5 \times 10^{-6}$ (measured power approximately $63 \mathrm{pW}$ ), comparable to previously reported results [5]. The converted power is linearly related to the pump and idler powers in 135 our experiments, as predicted by theory for sum and difference frequency generation [10]. It is worth pointing out that in our case, unlike the earlier experiments, a large number of NIR signals are obtained in the conversion process, corresponding to each THz idler mode from the QCL (see highlighted modes iiii) [5]. The low power of the up-converted modes could be improved by using double metal QCL waveguides [6], few mode QCLs with higher spectral power density, or by using optical amplification

140 techniques. Furthermore, the use of coherent detection techniques can help overcome the low power of these signals for both $\mathrm{THz}$ characterization and telecoms applications [9].

We now study the properties of this process by measuring both sideband power $\left(P_{\mathrm{s}}\right)$ and the transmitted pump power $\left(P_{\mathrm{p}}\right)$ to calculate the conversion efficiency $\left(P_{\mathrm{s}} / P_{\mathrm{p}}\right)$. This parameter can be directly compared between simulations and experiments, as is presented in Figure 2. Specifically, we can isolate each

145 individual mode up-converted from the THz to NIR to study the influence of phase matched pump frequency as a function of idler frequency, shown in Figure 2(a). Each NIR signal frequency has a distinct PM frequency, which makes it possible to study the dispersion of the nonlinear process. The inset of Figure 2a shows the fitted PM pump frequency as a function of $\mathrm{THz}$ (idler) mode frequency. An approximately linear relationship is observed between the THz mode frequency and the PM NIR pump, with a $d f_{\mathrm{p}} / d f_{\mathrm{i}}$ of $\sim 27$. This plot, and its slope, can be directly related to the group indices of each of the three waves at the phase matched frequency. Taking the derivative of equation (1) for sum frequency generation with respect to $f_{\mathrm{i}}$, results in the equation;

$$
\frac{d f_{p}}{d f_{i}}=\frac{n_{i}^{g}-n_{s}^{g}}{n_{s}^{g}-n_{p}^{g}}
$$


Where $n_{\mathrm{s}}^{\mathrm{g}}, n_{\mathrm{p}}^{\mathrm{g}} n_{\mathrm{i}}^{\mathrm{g}}$ are the group indices of the signal, pump and idler waves respectively. In general equation (6) should lead to nonlinear behavior in Figure 2a. However, the relatively large difference between $\mathrm{n}^{g}{ }_{i}\left(\sim 3.87\right.$ - measured from mode spacing) and $\mathrm{n}_{s}^{g}(\sim 3.6)$, means that equation (6) is relatively insensitive to small perturbations in $\mathrm{n}_{i}^{g}$ or $\mathrm{n}_{s}^{g}$ (such as those seen in simulations). Furthermore, the dispersion of GaAs in the near infrared is almost linear, meaning that $\mathrm{n}_{s}^{g}$ and $\mathrm{n}_{p}^{g}$ are similar and show comparable frequency dependence, so equation 6 is approximately constant. This is in general agreement 160 with simulations, where a small nonlinearity is observed due to the gain induced change in refractive index. This calculation can provide realistic limits to the frequency range $\left(\Delta f_{\mathrm{i}}\right)$ of terahertz modes which can be phase matched simultaneously using a fixed frequency pump laser. As the behavior of Figure 2a is approximately linear we can write $\Delta f_{\mathrm{i}}=\Delta f_{\mathrm{p}} \times\left(1 / d f_{\mathrm{p}} / d f_{\mathrm{i}}\right)$ and calculate that $\Delta f_{\mathrm{i}}=220 \mathrm{GHz}$. Equation 6 shows that this bandwidth is limited by the difference in group indices between the three waves [24]notably between the $\mathrm{THz}$ and infrared waves.
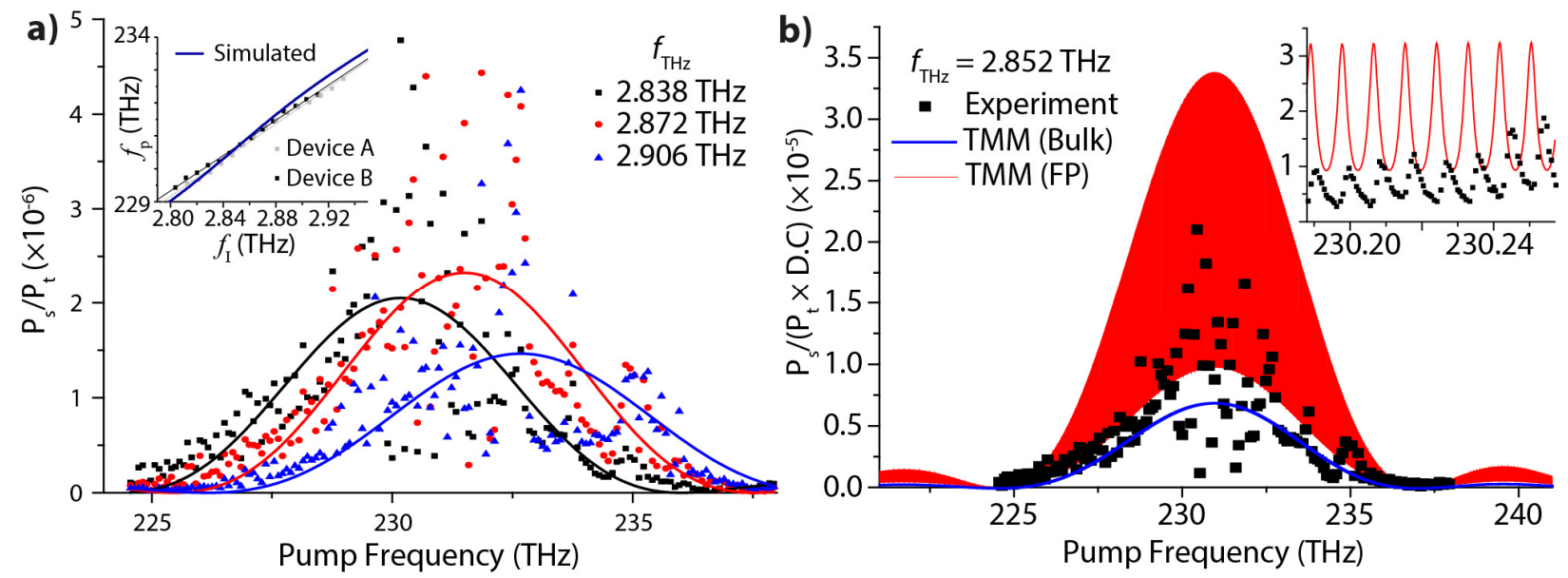

Figure 2. Experimental and simulated PM data. a) Measured efficiency data for three NIR sideband frequencies from Device A, with a frequency spacing $\Delta f=90 \mathrm{GHz}$, each fitted using a sinc ${ }^{2}$ function (solid lines). The three sinc ${ }^{2}$ fits shown reveal a full width half maximum PM bandwidth of $\Delta f_{\mathrm{p}}=5.985 \mathrm{THz}$. Inset: phase matched pump frequency as a function of idler frequency for devices A, B and simulations. b) Comparison between PM efficiency curves from TMM calculations and experiments. Note the efficiency has been normlised to peak power, accounting for the duty cycle. Inset shows high-resolution experimental data and TMM calculation of etalon fringes, measured for $\Delta f \sim 0.65 \mathrm{GHz}$. 
We note the scattering on the experimental results presented in Figure 2(a). This is a consequence of the cavity effect on the nonlinear process, and can be explained by comparing with the TMM results, shown 175 in Figure 2(b). Due to the high refractive index of GaAs and the presence of cleaved facets, the laser cavity produces etalon interference (reflectivity on each facet $\sim 0.29$, etalon reflectivity $\sim 0.7$ ) on the NIR pump and signal waves. Numerical results reveal etalon-like fringes [25] in the efficiency curve and a significant enhancement in signal (i.e. the sideband) power compared to the results for the bulk material without a cavity. These fringes are clearly resolved in experiments by measuring sideband conversion 180 efficiency over a narrow pump frequency range (Figure 2(a) inset). The influence of the cavity can be seen more clearly by comparing raw signal power (not efficiency) between simulations and experiments. Figure 3(a) shows a simulation of the output power relative to the input pump wave, revealing a significant enhancement in signal power compared with the bulk. Moreover, the feedback introduces an additional modulation to the $\operatorname{sinc}^{2}$-like PM curve (shown for the sum frequency signal). This modulation 185 is comparable to the product of the cavity transmission at the pump and signal frequencies $\left(T\left(f_{\mathrm{p}}\right) \times T\left(f_{\mathrm{s}}\right)\right)$; suggesting that the nonlinear process is enhanced by feedback on pump and signal waves. The properties of this interference effect are clearly seen in Figure 3(b) - where the power shows both single and double etalon fringes depending on the choice of pump frequency range. These effects can be observed experimentally by instead measuring different THz modes as $f_{\mathrm{NIR}}$ is finely tuned (Figure $3(\mathrm{c})$ )). The results 190 show similar double interference fringes, validating the TMM calculations. Crucially - our results show that up-conversion inside a THz laser is strongly enhanced by cavity feedback, as long as the pump frequency is accurately chosen. 

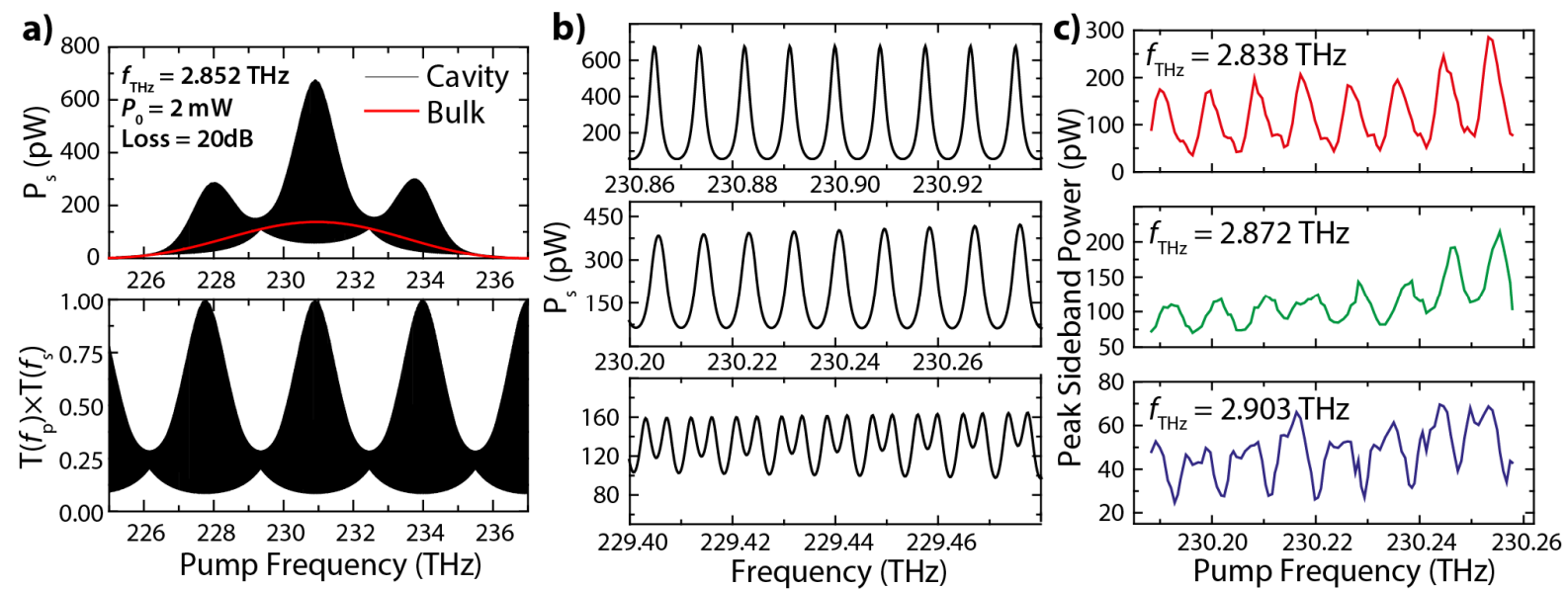

Figure 3. Sideband power as a function of pump frequency. a) Numerically calculated sideband power and the numerically calculated product between pump transmission $\left(T\left(f_{\mathrm{p}}\right)\right)$ and signal transmission $\left(T\left(f_{\mathrm{s}}\right)\right)$, b) etalon patterns generated within three different pump frequency ranges, c) measured experimental data from the three studied THz modes of device $A$.

In this letter, we have demonstrated multi-mode up-conversion of THz waves inside a QCL surfaceplasmon waveguide. Conversion efficiencies for this nonlinear mixing process show that each upconverted NIR signal has a distinct PM curve. A THz idler bandwidth of $220 \mathrm{GHz}$ can be measured using a single pump frequency; a span which could be further expanded with dispersion management techniques. The generated signal power is subject to the resonant properties of the etalon; by suitably choosing an appropriate resonant idler frequency it is possible to significantly enhance the measured conversion efficiency. This suggests that intra-cavity up-conversion offers an appealing route to both characterising THz sources, and applications in frequency conversion for both multicasting and demultiplexing.

Word Count; 3935/4375 (3500/4*5)

Text; 2654

Equations; 81 
210 Figures; 1200

Page 11 


\section{Acknowledgements}

The authors gratefully acknowledge EPSRC First Grant EP/ G064504/1 and funding from HMGCC. T. G. Folland acknowledges funding from the EPSRC North-West Nanoscience Doctoral Training Centre (Grant EP/G03737X/1). The authors would also like to thank David Madsen of DM Optics for loan of an optical spectrum analyser.

\section{References}

1. G. P. Agrawal, "Population pulsations and nondegenerate four-wave mixing in semiconductor lasers and amplifiers," J. Opt. Soc. Am. B 5, 147 (1988).

2. J. Madéo, P. Cavalié, J. R. Freeman, N. Jukam, J. Maysonnave, K. Maussang, H. E. Beere, D. A. Ritchie, C. Sirtori, J. Tignon, and S. S. Dhillon, "All-optical wavelength shifting in a semiconductor laser using resonant nonlinearities," Nat. Photonics 6, 519-524 (2012).

3. M. A. Belkin, F. Capasso, A. Belyanin, D. L. Sivco, A. Y. Cho, D. C. Oakley, C. J. Vineis, and G. W. Turner, "Terahertz quantum-cascade-laser source based on intracavity difference-frequency generation," Nat. Photonics 1, 288-292 (2007).

225 4. Q. Y. Lu, N. Bandyopadhyay, S. Slivken, Y. Bai, and M. Razeghi, "Widely tuned room temperature terahertz quantum cascade laser sources based on difference-frequency generation," Appl. Phys. Lett. 101, 251121 (2012).

5. S. S. Dhillon, C. Sirtori, S. Barbieri, A. de Rossi, M. Calligaro, H. E. Beere, and D. A. Ritchie, "THz sideband generation at telecom wavelengths in a GaAs-based quantum cascade laser," Appl. Phys. Lett. 87, 71101 (2005).

6. S. S. Dhillon, C. Sirtori, J. Alton, S. Barbieri, A. de Rossi, H. E. Beere, and D. A. Ritchie, "Terahertz transfer onto a telecom optical carrier," Nat. Photonics 1, 411-415 (2007).

7. S. Chakraborty, "Terahertz Mixer and Optical Fiber Coupled Terahertz Mixer," U.S. patent US20150248047 A1 (2015).

235 8. T. Folland, A. Ramos - Pulido, O. Marshall, H. Beere, D. Ritchie, and S. Chakraborty, "Highaccuracy heterodyne detection of $\mathrm{THz}$ radiation exploiting telecommunication technologies," in CLEO: 2015 (OSA, 2015), p. STu4H.3.

9. T. G. Folland, O. P. Marshall, H. E. Beere, D. A. Ritchie, and S. Chakraborty, "Coherent detection of THz laser signals in optical fiber systems," Opt. Express 25, 25566 (2017).

240 10. A. Yariv and P. Yeh, Photonics: Optical Electronics in Modern Communications (Oxford University, 2007).

11. V. Berger and C. Sirtori, "Nonlinear phase matching in THz semiconductor waveguides," Semicond. Sci. Technol. 19, 964-970 (2004).

12. V. D'Auria, S. Fornaro, A. Porzio, E. A. Sete, and S. Solimeno, "Fine tuning of a triply resonant 
B 91, 309-314 (2008).

13. D. V Strekalov, A. S. Kowligy, Y.-P. Huang, and P. Kumar, "Optical sum-frequency generation in a whispering-gallery-mode resonator," New J. Phys. New J. Phys. 16, 53025-53025 (2014).

14. S. Barbieri, M. Ravaro, P. Gellie, G. Santarelli, C. Manquest, C. Sirtori, S. P. Khanna, E. H. Linfield, and A. G. Davies, "Coherent sampling of active mode-locked terahertz quantum cascade lasers and frequency synthesis," Nat. Photonics 5, 306-313 (2011).

15. A. J. Seeds, M. J. Fice, K. Balakier, M. Natrella, O. Mitrofanov, M. Lamponi, M. Chtioui, F. van Dijk, M. Pepper, G. Aeppli, A. G. Davies, P. Dean, E. Linfield, and C. C. Renaud, "Coherent terahertz photonics.," Opt. Express 21, 22988-3000 (2013).

255 16. D. B. Anderson and J. T. Boyd, "Wideband CO2 Laser Second Harmonic Generation Phase Matched in GaAs Thin Film Waveguides," Appl. Phys. Lett. 19, 266 (1971).

17. L. Yan, A. E. Willner, X. Wu, A. Yi, A. Bogoni, Z.-Y. Chen, and H.-Y. Jiang, "All-Optical Signal Processing for UltraHigh Speed Optical Systems and Networks," J. Light. Technol. 30, 3760-3770 (2012).

260 18. M. Khairuzzaman, "Digitally Selected Electronically Switchable Terahertz-over-Fibre," PhD Thesis, The University of Manchester (2014).

19. J.-J. Li, Z.-Y. Li, and D.-Z. Zhang, "Second harmonic generation in one-dimensional nonlinear photonic crystals solved by the transfer matrix method," Phys. Rev. E 75, 56606 (2007).

20. M.-L. Ren and Z.-Y. Li, "Enhanced nonlinear frequency conversion in defective nonlinear photonic crystals with designed polarization distribution," J. Opt. Soc. Am. B 27, 1551 (2010).

21. T. G. Folland, L. Hua, and S. Chakraborty, "Threshold gain in aperiodic lattice lasers," Opt. Express 24, 30024-30030 (2016).

22. T. Skauli, P. S. Kuo, K. L. Vodopyanov, T. J. Pinguet, O. Levi, L. A. Eyres, J. S. Harris, M. M. Fejer, B. Gerard, L. Becouarn, and E. Lallier, "Improved dispersion relations for GaAs and applications to nonlinear optics," J. Appl. Phys. 94, 6447 (2003).

23. D. Burghoff, T.-Y. Kao, N. Han, C. W. I. Chan, X. Cai, Y. Yang, D. J. Hayton, J.-R. Gao, J. L. Reno, and Q. Hu, "Terahertz laser frequency combs," Nat. Photonics 8, 462-467 (2014).

24. T. G. Folland, "Frequency Control of Terahertz Quantum Cascade Lasers: Theory and Measurement," PhD Thesis, The University of Manchester (2017).

275 25. S. Chakraborty, D. G. Hasko, and R. J. Mears, "Aperiodic lattices in a high refractive index contrast system for photonic bandgap engineering," Microelectron. Eng. 73-74, 392-396 (2004). 
a) NIR Carrier $\mathrm{PC} \quad \mathrm{THz} Q \mathrm{QCL}$
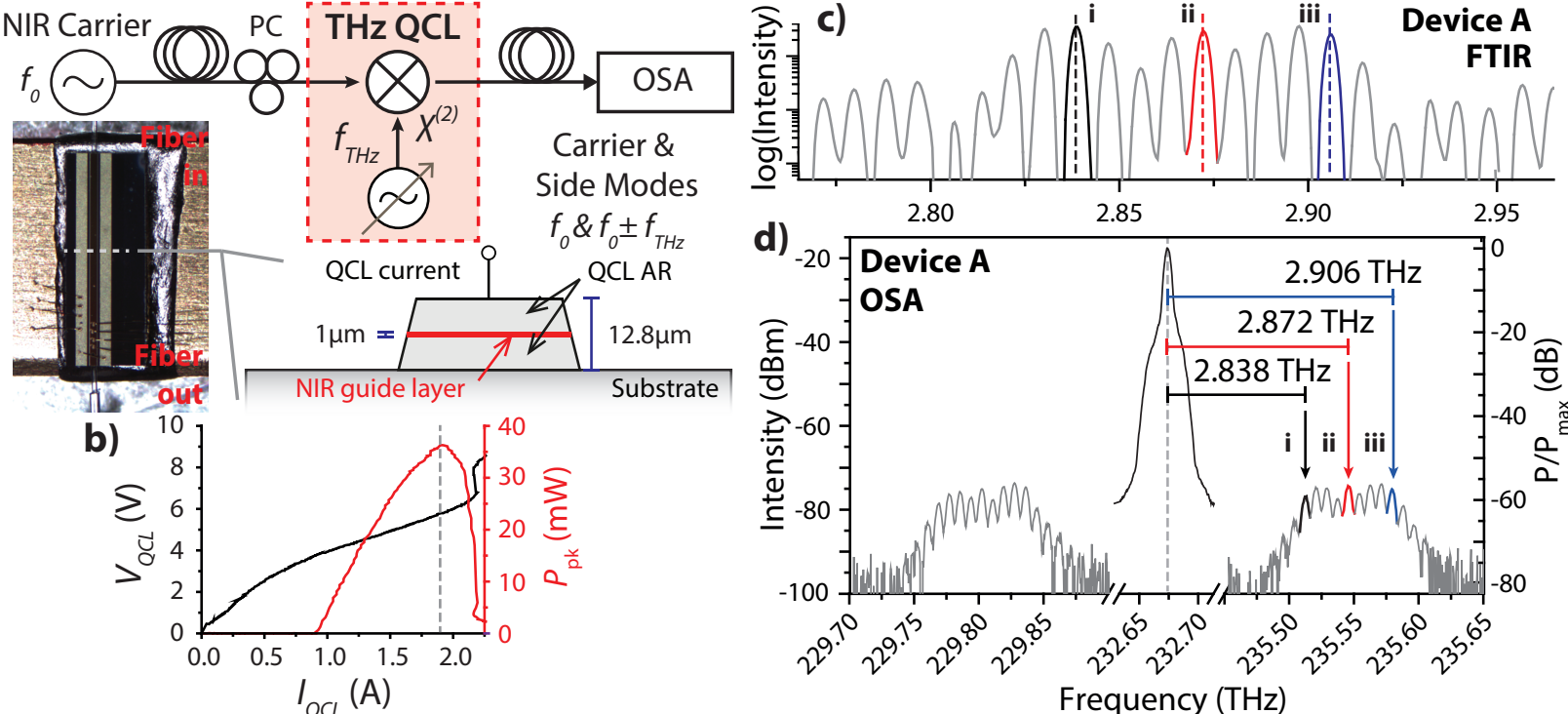

Frequency (THz) 


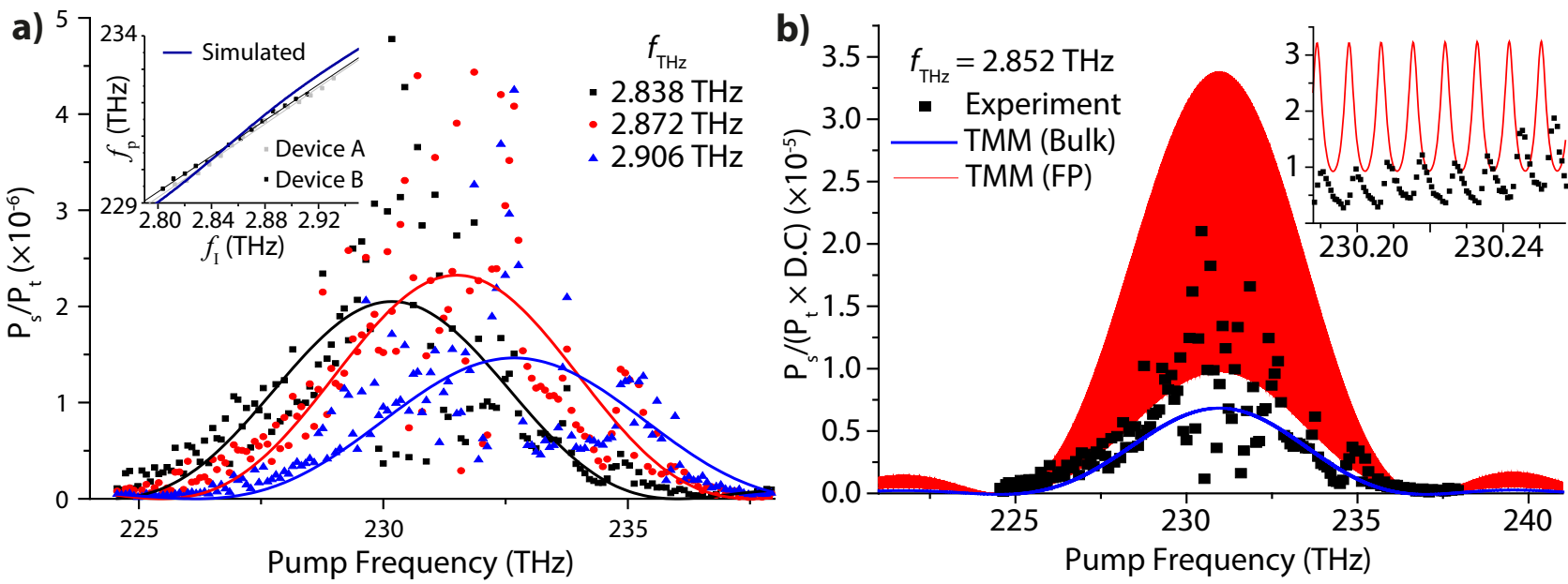


a)

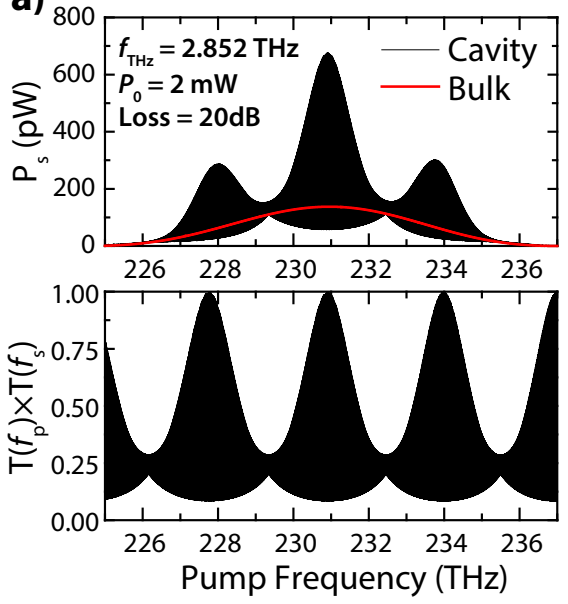

b)

b)

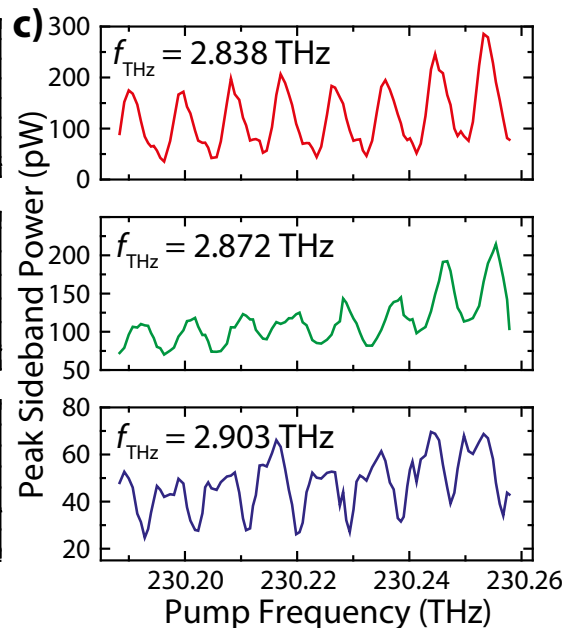

